S. B. BANK

KODAI MATH. J.

9 (1886), 225-240

\title{
THREE RESULTS IN THE VALUE-DISTRIBUTION THEORY OF SOLUTIONS OF LINEAR DIFFERENTIAL EQUATIONS ${ }^{(1)}$
}

\author{
By STEven. B. BANK
}

\begin{abstract}
For three different classes of equations of the form $f^{\prime \prime}+A(z) f=0$, where $A(z)$ is entire, we develop new information about the distribution of zeros of all solutions $f \not \equiv 0$, and thereby obtain a complete value-distribution theory for all solutions.
\end{abstract}

1. Introduction: In [1], we investigated the value-distribution of solutions $f \not \equiv$ of an equation of the form,

$$
f^{\prime \prime}+A(z) f=0,
$$

where $A(z)$ is an arbitrary entire function. Roughly speaking, it was shown that if one possesses sufficient information on the counting functions for the zeros of all solutions of (1), then for any solution $f \neq 0$ of (1), one can determine all polynomials $P\left(z, u_{0}, \cdots, u_{n}\right)$, having meromorphic coefficients of slower growth than $f$, for which the function,

$$
h(z)=P\left(z, f(z), f^{\prime}(z), \cdots, f^{(n)}(z)\right),
$$

is either identically zero, or has the property that the counting function $\bar{N}(r, 1 / h)$ for its distinct zeros is of slower growth than $f$. (Of course, in view of (1), the function $h$ in (2) will also be given by a first-order differential polynomial $P^{*}\left(z, f(z), f^{\prime}(z)\right)$, and so attention can be restricted to polynomials $P(z, u, v)$ in two indeterminates.) It was shown in [1] that the existence and form of these "special" polynomials for a solution of a given equation (1), depends heavily on whether (1) possesses none, one, or two linearly independent solutions $f \not \equiv 0$ having the property that ${ }^{"} \bar{N}(r, 1 / f)$ grows slower than $f$. (Examples illustrating each of the three possibilities can be found in [1].)

To take a concrete situation, if $A(z)$ is a transcendental entire function of finite order of growth, and if $f \not \equiv 0$ is any solution of (1), then the result in $[1$; Corollary 3, p. 510] will determine all the polynomials $P(z, u, v)$ of positive total

(1) This research was supported in part by the National Science Foundation (DMS. 8420561).

Received October 24, 1985 
degree in the indeterminates $u$ and $v$, having arbitrary meromorphic coefficients of finite order, for which the function,

$$
h(z)=P\left(z, f(z), f^{\prime}(z)\right),
$$

is either identically zero, or has the property that the exponent of convergence (denoted $\bar{\lambda}(h)$ ) of its sequence of distinct zeros is finite. To apply this result, we must know whether (1) possesses none, one, or two linearly independent solutions $f \not \equiv 0$ for which $\bar{\lambda}(f)<\infty$. Some general results already exist to help decide this matter in certain cases where the order of $A(z)$ is small or is a positive integer. For example, in [2] it was shown that if the order of $A(z)$ is less than $1 / 2$, then for any two linearly independent solutions $f_{1}, f_{2}$ of (1), we have,

$$
\max \left\{\bar{\lambda}\left(f_{1}\right), \bar{\lambda}\left(f_{2}\right)\right\}=\infty \text {. }
$$

(Recent preprints by L.-C. Shen and by J. Rossi indicate that the conclusion (4) also holds when the order of $A(z)$ equals $1 / 2$.)

As a second example, it was shown in [4] that the same conclusion (4) holds whenever the coefficient function $A(z)$ has the form,

$$
A(z)=\sum_{j=1}^{n} B_{j}(z) e^{Q_{j}(z)}+Q(z),
$$

where (i) $Q_{1}, \cdots, Q_{n}$ are nonconstant polynomials whose respective degrees $d_{1}, \cdots, d_{n}$ satisfy $\operatorname{deg}\left(Q_{i}-Q_{j}\right)=\max \left\{d_{\imath}, d_{j}\right\}$ for $i \neq j$; (ii) each $B$, is an entire function, not identically zero, of order less that $d_{j}$; (iii) either $Q \equiv 0$ or $Q$ is a polynomial of degree less than $2 d-2$, where $d=\max \left\{d_{1}, \cdots, d_{n}\right\}$. (Of course, the order of $A(z)$ is the positive integer $d$. )

In our first result of the present paper, we consider a class of equations (1) where the order of $A(z)$ can be any nonnegative real number, and we show that if the equation possesses a solution $f_{1} \not \equiv 0$ satisfying $\bar{\lambda}\left(f_{1}\right)<\infty$, and which is of a certain form, then for any solution $f_{2}$ which is linearly independent with $f_{1}$, we must have $\bar{\lambda}\left(f_{2}\right)=\infty$. (Thus for this class of equations, sufficient information now exists to apply the result in $[1$; Corollary 3 , Parts (b), (d)], to determine for any solution $f \not \equiv 0$, the class of all $P(z, u, v)$ having meromorphic coefficients of finite order, for which $\bar{\lambda}(h)<\infty$ where $h$ is given in (3).) We will prove the following theorem in $\S 3$ :

THEOREM 1. Let $g(z)$ be an entire function of finite order for which there exist real numbers $a$ and $b$ such that $g(z)$ is positive on both $(a,+\infty)$ and $(-\infty, b)$, and assume that for every $\alpha>0$, we have

(A) $g(r) / r^{\alpha} \rightarrow+\infty$ as $r \rightarrow+\infty$ through real values, and

(B) $g(s) /|s|^{\alpha} \rightarrow+\infty$ as $s \rightarrow-\infty$ through real values.

Let $G(z)$ be an entire function of finite order, which is real for real $z$, and which has no zeros on an infinite strip $|\operatorname{Im} z|<\delta$, for some $\delta>0$. Then, if $f=G e^{g}$ satisfies an equation $f^{\prime \prime}+A f=0$, where $A$ is entire, we have $\bar{\lambda}\left(f_{1}\right)=\infty$ for any 
solution $f_{1} \neq 0$ of this equatıon which is not a constant multiple of $f$.

We make four brief remarks concerning this theorem. First, there is no lack of examples to which this theorem will apply, since any function of the form $e^{g}$, where $g$ is entire, satisfies an equation of the form (1), and, in addition, if $g$ is an even, transcendental, entire function of finite order, whose power series expansion around $z=0$ has all nonnegative coefficients, then $g$ will also satisfy the conditions (A) and (B) in the theorem. Second, it is easy to see ( $\$ 4$ below) that the class of equations treated in Theorem 1, contains coefficient functions of any preassigned finite order. Third, it is tempting to try to strengthen Theorem 1 by possibly deleting one of the conditions (A) or (B). However, for $g(z)=e^{z}-(z / 2)$ (which satisfies condition (A), but not (B)), it is easy to see that $f=e^{g}$ satisfies the same equation (1) as $f_{1}=\exp \left(-\left(e^{z}+(z / 2)\right)\right)$, namely where $A(z)=-\left(e^{2 z}+(1 / 4)\right)$, and it is clear that $f$ and $f_{1}$ are linearly independent, and $\bar{\lambda}\left(f_{1}\right)=0$. Finally, we remark that there are cases of coefficient functions of the form (5), where the condition (iii) on $Q$ mentioned earlier is violated (so that the result of [4] is not applicable), but which can be treated by Theorem 1. An example is furnished by the function $f(z)=\exp \left(e^{z}+e^{-z}\right)$ which satisfies (1) where

$$
A(z)=-e^{2 z}-e^{z}-e^{-z}-e^{-2 z}+2 .
$$

(Here $Q \equiv 2$, and is not of degree less than $2 d-2$ since $d=1$.). However, since $g(z)=e^{z}+e^{-z}$ satisfies (A) and (B) of Theorem 1 , we can conclude that $\bar{\lambda}\left(f_{1}\right)=\infty$ for every solution $f_{1} \not \equiv 0$ which is not a constant multiple of $f$.

Our second result concerns the value-distribution theory for the solutions of a class of equations (1) where $A(z)$ is a periodic entire function of the form $B\left(e^{\alpha z}\right)$, where $B(\zeta)$ is a rational function, and where $\alpha$ is a nonzero constant. It was proved in [3; Theorem 3], that if the rational function $B(\zeta)$ has poles of odd order at both $\zeta=0$ and $\zeta=\infty$, then for any solution $f \not \equiv 0$ of (1), we have $\bar{\lambda}(f)=\infty$. (This class of equations contains the Mathieu equation.) This result provides sufficient information to apply $[1$; Corollary $3(\mathrm{a})]$ to conclude that for any solution $f \not \equiv$, and any polynomial $P(z, u, v)$ in $u$ and $v$, of positive total degree, having meromorphic coefficients of finite order, the function $h$ in (3) satisfies the conditions $h \not \equiv, \bar{\lambda}(h)=\infty$. However, as indicated in [1; Theorem 2], the stronger the information we possess concerning the zeros of the solutions of an equation (1), the stronger will be the value-distribution theory for the solutions that we obtain from [1; Theorem 2]. In our second result, we make two substantial improvements in [3; Theorem 3], and thereby obtain a stronger value-distribution theory for a broader class of equations. First, we prove that the conclusion $\bar{\lambda}(f)=\infty$ for every solution $f \not \equiv 0$, can be replaced by the stronger conclusion,

$$
\log ^{+} \bar{N}(r, 1 / f) \neq o(r) \quad \text { as } \quad r \rightarrow+\infty .
$$

For our second improvement, we show that instead of requiring both of the 
poles of $B(\zeta)$ at $\zeta=0$ and $\zeta=\infty$ to be of odd order, our stronger conclusion (7) will hold for all solutions $f \not \equiv 0$ when at least one of these poles is of odd order. (The example, $f(z)=\exp \left(e^{z}+e^{-z}\right)$ which satisfies (1) where $A(z)$ is given by (6), shows that when the poles of $B(\zeta)$ at $\zeta=0, \infty$ are both of even order, the conclusion (7) can fail for some solution $f \neq 0$ of (1). However, there are examples (see $\$ 9$ ) where the poles of $B(\zeta)$ are both of even order, and $(7)$ does hold for all solutions $f \neq 0$ of $(1)$. In addition, in the case where $B(\zeta)$ has a pole at only one of the points $\zeta=0, \infty$, we have seen earlier that the conclusion (7) can fail to hold for some solution $f \not \equiv 0$, since $f(z)=\exp \left(e^{z}-(z / 2)\right)$ satisfies (1) where $A(z)=-\left(e^{2 z}+(1 / 4)\right)$. However, in $\S 9$ we show that there are examples of such equations where $(7)$ holds for all solutions $f \not \equiv 0$.) We will prove the following theorem in $\S 8$ :

THEOREM 2. Let $B(\zeta)$ be a rational function which is analytic on $0<|\zeta|<\infty$, and which has poles at both $\zeta=0$ and $\zeta=\infty$, and assume that at least one of these poles is of odd order. Let $\alpha$ be a nonzero constant, and set $A(z)=B\left(e^{\alpha z}\right)$. Then

(A) For any solution $f \not \equiv 0$ of $f^{\prime \prime}+A(z) f=0$, the conclusion (7) holds.

(B) Let $\Delta(r)$ be any unbounded increasing function on $(1,+\infty)$ with the property that as $r \rightarrow+\infty$ outside a possible exceptional set of finite measure, we have $r=0(\Delta(r))$ and $\log ^{+} \Delta(r)=o(r)$. Let $P(z, u, v)$ be any polynomial in $u$ and $v$, of positive total degree, whose coefficients are any meromorphic functions $a(z)$ satisfying the condition that $T(r, a)=0(\Delta(r))$ as $r \rightarrow+\infty$ outside a possible exceptional set of finite measure. Then, if $f \not \equiv 0$ is any solution of $f^{\prime \prime}+A(z) f=0$, the function $h(z)=P\left(z, f(z), f^{\prime}(z)\right)$ cannot be identically zero, and $h(z)$ cannot have the property that $\bar{N}(r, 1 / h)=0(\Delta(r))$ as $r \rightarrow+\infty$ outside a possible exceptional set of finite measure.

We remark that convenient choices for $\Delta(r)$ in Part (B) such as $\Delta(r)=$ $\exp \left(r(\log r)^{-1}\right)$ or $\Delta(r)=\exp \left(r^{\alpha}\right)$ with $0<\alpha<1$, allow us to examine polynomials $P(z, u, v)$ having coefficients of infinite order. In addition, we point out here that the conclusion (7) of Part (A) cannot be greatly improved, since it follows easily from [1; Lemma 4.1 (a), p. 519] that for every solution $f \neq 0$, we have $\log ^{+} N(r, 1 / f)=0(r)$ as $r \rightarrow+\infty$. (We remark here that various classes of linear differential equations with periodic coefficients have been treated by Frei [5], Ozawa [9], and Wittich [13].)

Our final result is also an improvement of a previous result, namely the value-distribution theory given in [1; Theorem 2] for the solutions of (1) in the case when $A(z)$ is a nonconstant polynomial of degree $n$. In this case, the solutions $f \not \equiv 0$ of $(1)$ are of $\operatorname{order}(n+2) / 2$ (see $[11 ;$ p. 106] or $[12 ;$ p. 281]), and the result in [1; Theorem 2] determines the polynomials $P(z, u, v)$ in $u$ and $v$, of positive total degree, having meromorphic coefficients of order less than $(n+2) / 2$, for which the function $h(z)$ given by (3) is either identically zero, or has the property that $\bar{\lambda}(h)<(n+2) / 2$. However, one can permit more general coefficients for $P(z, u, v)$, namely all meromorphic functions $a(z)$ having the following property: 


$$
T(r, a)=o\left(r^{(n+2) / 2}\right) \quad \text { as } \quad r \rightarrow+\infty .
$$

In our final theorem, we determine all such $P(z, u, v)$ for which either $h(z) \equiv 0$ or

$$
\bar{N}(r, 1 / h)=o\left(r^{(n+2) / 2}\right) \quad \text { as } \quad r \rightarrow+\infty .
$$

The proof (which is given in $\S 10$ ) consists simply in showing that the hypothesis of $[1$; Theorem 4$]$ is satisfied for the differential field consisting of all meromorphic functions satisfying (8). This follows very easily from classical results of Hille, Nevanlinna, and Wittich (see [12; pp. 282-283]), and also from a new non-asymptotic approach due to $\mathrm{R}$. Kaufman [7; Theorem 2]. Before stating the theorem, we remark that there is a simple algorithm $[1 ; \S 7(\mathrm{a})]$ to determine for any equation (1) where $A(z)$ is a polynomial, whether (1) possesses a solution having only finitely many zeros, and to determine the solution if it exists.

Theorem 3. Let $A(z)$ be a polynomial of degree $n \geqq 1$, and let $H_{n}$ denote the field of all meromorphic functions $a(z)$ satisfying (8). Let $P(z, u, v)$ be any polynomial in $u$ and $v$, of positive total degree in $u$ and $v$, having coefficients in $H_{n}$. Then:

(A) If all solutions $f \not \equiv 0$ of (1) have infinitely many zeros, then for any solution $f \not 0$ of (1), the function $h(z)$ given by (3) is not identically zero, and $h$ cannot satisfy (9).

(B) Assume that (1) possesses a solution $f_{0} \neq 0$ having only finitely many zeros, and set $R_{0}=-f_{0}^{\prime} / f_{0}$. Then:

(a) For any solution $f \not 0$ of (1) which is not a constant multiple of $f_{0}$, the function $h(z)$ given by (3) is not identically zero. In addition, (9) holds if and only if $P(z, u, v)$ has the special form

$$
P(z, u, v)=G(z)\left(v+R_{0}(z) u\right)^{m},
$$

for some positive integer $m$, and some function $G(z) \equiv \equiv$ belonging to $H_{n}$.

(b) For any solution $f \neq 0$ of (1) which is a constant multiple of $f_{0}$, the function $h(z)$ given by (3) is identically zero if and only if $P(z, u, v)$ has the form,

$$
P(z, u, v)=Q(z, u, v)\left(v+R_{0}(z) u\right),
$$

for some polynomial $Q(z, u, v)$ in $u$ and $v$ with coefficients belonging to $H_{n}$ (and some coefficient not identically zero). In addition, if $h(z) \neq 0$, then (9) holds if and only if $P(z, u, v)$ has the form,

$$
P(z, u, v)=Q(z, u, v)\left(v+R_{0}(z) u\right)+E(z, u, v),
$$

for some polynomials $Q$ and $E$ in $u$ and $v$, having coefficients in $H_{n}$, and where all terms in $E(z, u, v)$ have the same total degree in $u$ and $v$, and where $E(z, 1$, $\left.-R_{0}(z)\right) \not \equiv 0$.

Finally, the author would like to acknowledge valuable conversations with W. K. Hayman during his visit to Urbana in May, 1985. 
2. LEMMA A. Let $g(z)$ be an entire function of finte order for which there is a real number $b$ such that $\operatorname{Re}(g(r))>0$ on $(b,+\infty)$, and assume that for every $\alpha>0$,

$$
r^{-\alpha}(\operatorname{Re}(g(r))) \longrightarrow+\infty \text { as } r \rightarrow+\infty \text { through real values. }
$$

Let $G(z)$ be an entire function of finite order which has no zeros on a set of the form, $|\operatorname{Im}(z)|<\delta, \operatorname{Re}(z)>a$, where $\delta>0$ and $a \geqq-\infty$, and assume that $f=G e^{g}$ is a solution of an equation $f^{\prime \prime}+A(z) f=0$, where $A(z)$ is entire. Then, if this equation possesses a solution $f_{1} \not \equiv 0$ satisfying $\tilde{\lambda}\left(f_{1}\right)<\infty$, and such that $f_{1}$ is not a constant multiple of $f$, the following hold:

(a) $f_{1}=H e^{-g}$ for some entire function $H(z)$ of finite order.

(b) For all $r>a$,

$$
H(r) / G(r)=-K e^{2 g(r)} \int_{r}^{+\infty}\left(e^{-2 g(t)} /(G(t))^{2}\right) d t,
$$

where $K$ is the Wronskian of $f$ and $f_{1}$.

Proof. Since $A=-f^{\prime \prime} / f$, we clearly have,

$$
A=-\left(\left(g^{\prime}\right)^{2}+g^{\prime \prime}+2 g^{\prime}\left(G^{\prime} / G\right)+\left(G^{\prime \prime} / G\right)\right),
$$

which shows that $A(z)$ is of finite order. If we set $E=f f_{1}$, it follows (see [3; Lemma $\mathrm{B}]$ ) that $E$ satisfies the equation,

$$
K^{2}-\left(E^{\prime}\right)^{2}+2 E E^{\prime \prime}+4 A E^{2}=0,
$$

for some constant $K \neq 0$, and the relation,

$$
T(r, E)=0(\bar{N}(r, 1 / E)+T(r, A)+\log r),
$$

as $r \rightarrow+\infty$ "nearly everywhere" (briefly, n. e., meaning outside a possible exceptional set of finite measure). From the hypothesis, we see from (17) (and [3; $\S 4(\mathrm{~A})]$ ) that $E$ is of finite order, and so the representation in Part (a) follows when we set $H=E / G$.

For Part (b), we observe first that since $G$ has no zeros on $|\operatorname{Im}(z)|<\delta$, $\operatorname{Re}(z)>a$, it follows from a standard minimum modulus estimate (see [10; p. 336]) that there are positive constants $r_{0}$ and $\beta$ such that

$$
|G(t)| \geqq \exp \left(-t^{\beta}\right) \quad \text { for all } t \geqq r_{0} .
$$

(We remark that when $a=-\infty$, we actually obtain

$$
|G(t)| \geqq \exp \left(-|t|^{\beta}\right) \text { for all real } t \text { satisfying }|t| \geqq r_{0},
$$

by $[10 ;$ p. 336].).

In view of the hypothesis (13), and the estimate (18), it easily follows that for all $r>a$, the integral, 


$$
F(r)=\int_{r}^{+\infty}\left(e^{-2 g(t)} /(G(t))^{2}\right) d t,
$$

converges, and represents a differentiable function on $(a,+\infty)$ with the properties,

$$
F^{\prime}(r) \equiv-e^{-2 g(r)} /(G(r))^{2} \text {, and } F(r) \rightarrow 0 \quad \text { as } r \rightarrow+\infty \text {. }
$$

Now, since $f$ and $f_{1}$ are both solutions of (1), we have by Abel's identity that the derivative of $f_{1} / f$ is $K / f^{2}$. Since $f=G e^{g}$ and $f_{1}=H e^{-g}$ (by Part (a)), we see that if we set $w=H / G$, then the derivative of $w(r) e^{-2 g(r)}$ is $-K F^{\prime}(r)$ by (21), and so there exists a constant $L$ such that,

$$
w(r) e^{-2 g(r)} \equiv-K F(r)+L \quad \text { on } \quad(a,+\infty) .
$$

We assert that $L=0$. We know from (21) that $F(r) \rightarrow 0$ as $r \rightarrow+\infty$. Since $H(z)$ is of finite order by Part (a), it follows from (18) that there are positive constants $r_{1}$ and $b$ such that

$$
|w(r)| \leqq \exp \left(r^{b}\right) \quad \text { for all } r>r_{1} .
$$

Thus in view of the hypothesis (13), the left-hand side of (22) approaches zero as $r \rightarrow+\infty$ so $L=0$. Thus (14) follows from (22), and so Lemma $A$ is proved.

3. Proof of Theorem 1. We assume the conclusion fails to hold, so there exists a solution $f_{1}$ which is not a constant multiple of $f$, and satisfying $\bar{\lambda}\left(f_{1}\right)<\infty$. Hence by Lemma A, we can write $f_{1}=H e^{-s}$ where $H(z)$ is an entire function of finite order, and (14) holds where $a=-\infty$. Since $a=-\infty$, the estimate (19) holds, and since $H(z)$ is of finite order, we see that there are positive constants $b$ and $r_{2}$ such that

$$
|H(s) / G(s)| \leqq \exp \left(|s|^{b}\right) \text { for all real } s \text { satisfying }|s| \geqq r_{2} .
$$

Now, by the hypothesis, $G$ and $g$ are real on the real axis, and so the integrand in (14) is a positive continuous function of $t$ on $(-\infty, \infty)$. Hence for $s<-r_{2}$,

$$
\left|\int_{s}^{+\infty}\left(e^{-2 g(t)} /(\vec{z} G(t))^{2}\right) d t\right| \geqq \int_{0}^{+\infty}\left(e^{-2 g(t)} /(G(t))^{2}\right) d t .
$$

Denoting by $K_{1}$ the right-hand side of (25), we have $K_{1}>0$, and from (14) and (24) we see that

$$
\exp \left(|s|^{b}\right) \geqq|K| K_{1} e^{2 g(s)} \quad \text { for all } s<-r_{2} .
$$

Of course, (26) is in direct contradiction to the hypothesis (B) for $\alpha=b$, proving the result.

4. Remark. In this section, we show that the class of equations (1) treated in Theorem 1 contains coefficient functions of any preassigned order. To this 
end, let $\sigma$ be any nonnegative real number. It is easy to construct (see [10; p. 326]) an even transcendental entire function $g$ of order $\sigma$ whose power series expansion around $z=0$ has all positive coefficients. Then $g$ satisfies (A) and (B) in Theorem 1. The function $f=e^{g}$ satisfies equation (1) with $A=-\left(\left(g^{\prime}\right)^{2}+g^{\prime \prime}\right)$. This relation shows that the order $\sigma_{1}$ of $A$ is equal to $\sigma$. (The inequality $\sigma_{1} \leqq \sigma$ is obvious, and the impossibility that $\sigma_{1}<\sigma$ follows immediately from a variant of Clunie's lemma [3; Lemma A, p. 4].)

5. We remark that the proofs of the results to be presented in $\S 5-7$ parallel very closely the proofs of the analogous results presented in [3].

LEMMA B. Let $B(\zeta)$ be a nonconstant rational function which is analytic on $0<|\zeta|<\infty$. Let $\alpha$ be a nonzero constant, and set $w=2 \pi i / \alpha$, and $A(z)=B\left(e^{\alpha z}\right)$. Let $f \not 0$ be a solution of (1) which satisfies the condition,

$$
\log ^{+} N(r, 1 / f)=o(r) \quad \text { as } r \rightarrow+\infty .
$$

Then, if the functions $f(z)$ and $f(z+w)$ are linearly dependent, the function $f(z)$ can be represented in the form,

$$
f(z)=\Psi\left(e^{\alpha z}\right) \exp \left(\sum_{j=q}^{m} d_{j} e^{\alpha \jmath z}+d z\right),
$$

where (i) $\Psi(\zeta)$ is a polynomial all of whose roots are simple and nonzero; (ii) $m$ and $q$ are integers with $m \geqq q$; (iii) $d, d_{q}, \cdots, d_{m}$ are complex constants such that $d_{j} \neq 0$ for some $j \neq 0$.

Proof. From the hypothesis that $f(z)$ and $f(z+w)$ are dependent, we can write $f(z)=e^{\beta z} U(z)$ where $U$ is entire and has period $w$. Thus $U(z)=G\left(e^{\alpha z}\right)$ where $G(\zeta)$ is analytic on $0<|\zeta|<\infty$, and from (1), it is easy to check that $G(\zeta)$ satisfies the equation

$$
\alpha^{2} \zeta^{2} G^{\prime \prime}+\zeta\left(2 \beta \alpha+\alpha^{2}\right) G^{\prime}+\left(B(\zeta)+\beta^{2}\right) G=0 .
$$

As in $[3 ;$ p. 9$]$, we first show that $G(\zeta)$ has only finitely many zeros on $0<|\zeta|$ $<\infty$. If we assume the contrary, the sequence of zeros of $G$ has a cluster point $\zeta^{*}$ at either 0 or $\infty$ (or both). If $\zeta^{*}=\infty$, then $G(\zeta)$ has an essential singularity at $\infty$, and by the Wiman-Valiron theory $[11 ; \mathrm{pp} .15,93-111]$ applied to (29), we can write $G(\zeta)$ in the form $\zeta^{m} \Phi(\zeta) u(\zeta)$, where $m$ is an integer, $\Phi(\zeta)$ is analytic and nonvanishing at $\infty$, and where $u(\zeta)$ is an entire transcendental function of finite order having infinitely many zeros. Letting $H(\zeta)$ denote the canonical product formed with the zeros of $u(\zeta)$, we have a representation $u(\zeta)$ $=H(\zeta) \exp (Q(\zeta))$ where $Q(\zeta)$ is a polynomial. Now from (29), the function $G_{1}=G e^{-Q}$ also satisfies a second-order linear differential equation with polynomial coefficients, and in view of the representation $G_{1}(\zeta)=\zeta^{m} \Phi(\zeta) H(\zeta)$, it again follows from the Wiman-Valiron theory that the entire transcendental function $H(\zeta)$ must have order $\delta>0$. Thus $\bar{\lambda}(u)=\delta>0$. Hence (see $[6 ;$ p. 25] or $[8 ;$ p. 27] or $[11$; 
p. 51]) the counting function for the zeros of $u$ satisfies

$$
\limsup _{t \rightarrow+\infty}((\log n(t, 1 / u)) / \log t)=\delta .
$$

Hence there exists a sequence $\left(t_{q}\right) \rightarrow+\infty$ such that

$$
\log n\left(t_{q}, 1 / u\right)>(\delta / 2) \log t_{q} \text { for all } q .
$$

Now, in view of the representation,

$$
f(z)=e^{\beta z} e^{\alpha m z} \Phi\left(e^{\alpha z}\right) u\left(e^{\alpha z}\right),
$$

and the fact that $\Phi(\zeta)$ is analytic and nowhere zero on a region $|\zeta|>r_{0}$ where $r_{0}>1$, it follows that if $\zeta_{0}$ is a zero of $u$ satisfying $\left|\zeta_{0}\right|>r_{0}$, then for any $z_{0}$ satisfying $e^{\alpha z_{0}}=\zeta_{0}$, we have $f\left(z_{0}\right)=0$. Let $\zeta_{1}, \zeta_{2}, \cdots$ be the zero-sequence of $u(\zeta)$, ordered by increasing modulus, and let $b$ be an index such that $\left|\zeta_{j}\right|>r_{0}$ for $j \geqq b$. For an index $q$, set $p=n\left(t_{q}, 1 / u\right)$ so that $\zeta_{1}, \cdots, \zeta_{p}$ are the zeros of $u$ in $|\zeta| \leqq t_{q}$. For $j \geqq b$, write $\zeta_{\jmath}=\left|\zeta_{j}\right| e^{i \theta_{j}}$ where $-\pi<\theta_{\jmath} \leqq \pi$, and set

$$
z_{j}=(1 / \alpha)\left(\log \left|\zeta_{j}\right|+\imath \theta_{j}\right)
$$

Thus $e^{\alpha z_{\jmath}}=\zeta_{\jmath}$, so by our previous observation, $z_{b+1}, \cdots, z_{p}$ are zeros of $f$, and each of these zeros lies in the disk $|z| \leqq|\alpha|^{-1}\left(\left(\log t_{q}\right)+\pi\right)$. Hence if $q$ is sufficiently large so that $t_{q}>e^{\pi}$, we have

$$
n\left(|\alpha|^{-1} 2\left(\log t_{q}\right), 1 / f\right) \geqq n\left(t_{q}, 1 / u\right)-b .
$$

Setting $s_{q}=|\alpha|^{-1} 2\left(\log t_{q}\right)$, we have $s_{q} \rightarrow+\infty$, and from (31), we obtain

$$
n\left(s_{q}, 1 / f\right)>e^{c s_{q}-h} \quad \text { where } \quad c=|\alpha| \delta / 4>0 .
$$

But this implies (see $[1 ;(10.17)$, p. 530]) that for all sufficiently large $q$,

$$
N\left(2 s_{q}, 1 / f\right)>(\log 2)\left(e^{c s_{q}}-b\right),
$$

which obviously contradicts our assumption (27) since $c>0$ and $\left(s_{q}\right) \rightarrow+\infty$. This shows that $\zeta^{*}$ cannot be $\infty$, and a similar argument applied to $G_{2}(t)=G(1 / t)$ in place of $G(\zeta)$ shows that $\zeta^{*}$ cannot be zero either. Thus $G(\zeta)$ has only finitely many zeros on $0<|\zeta|<\infty$, and each zero is simple since $f$ has only simple zeros. Letting $\Psi(\zeta)$ be the polynomial having these zeros, clearly $\varphi=G / \Psi$ is analytic and nowhere zero on $0<|\zeta|<\infty$. Hence $\varphi\left(e^{\alpha z}\right)$ is an entire function with no zeros, so is of the form $e^{v(z)}$, where $v(z)$ is entire. Clearly $v^{\prime}(z)$ has period $w$, and thus $v^{\prime}(z)=W\left(e^{\alpha z}\right)$ where $W(\zeta)$ is analytic on $0<|\zeta|<\infty$. Now since

$$
G^{\prime}(\zeta) / G(\zeta)=\left(\Psi^{\prime}(\zeta) / \Psi(\zeta)\right)+(\alpha \zeta)^{-1} W(\zeta)
$$

it follows easily from $(29)$ that $W(\zeta)$ satisfies a nonlinear Riccati equation with rational coefficients, and the Wiman-Valiron theory then shows that $W(\zeta)$ cannot have an essential singularity at either $\zeta=0$ or $\zeta=\infty$. Hence $W(\zeta)$ is rational, 
and so has the form,

$$
W(\zeta)=\zeta^{-k}\left(c_{n} \zeta^{n}+c_{n-1} \zeta^{n-1}+\cdots+c_{0}\right) .
$$

Integrating $v^{\prime}(z)=W\left(e^{\alpha z}\right)$ to find $v(z)$, the representation (28) follows immediately.

6. Lemma C. Let $A(z)$ be a nonconstant entire function of period $w$, and let $f \not 0$ be a solution of (1) which satisfies (27). Then, $f(z)$ and $f(z+2 w)$ are linearly dependent solutions of (1).

Proof. The conclusion is immediate if $f(z)$ and $f(z+w)$ are linearly dependent, so we may assume that $f(z)$ and $f(z+w)$ are linearly independent. Hence if we assume the conclusion is false, then the solutions $f_{1}=f, f_{2}(z)=$ $f(z+w)$, and $f_{3}(z)=f(z+2 w)$ are pairwise linearly independent, so by [1; Lemma 8.1, p. 523] we have n. e. as $r \rightarrow+\infty$,

$$
T\left(r, f_{1}\right)=0\left(\sum_{j=1}^{3} N\left(r, 1 / f_{j}\right)+T(r, A)\right) .
$$

Now, it is easy to see (e.g. [1; p. 530]) that for all sufficiently large $r$,

$$
N\left(r, 1 / f_{j}\right)=0\left((\log r) N\left(2 r, 1 / f_{1}\right)\right)
$$

for $j=1,2,3$, and since $A=-f_{1}^{\prime \prime} / f_{1}$, we have

$$
T(r, A)=0\left(\log T\left(r, f_{1}\right)\right) \quad \text { n.e. as } r \rightarrow+\infty .
$$

Hence from (39), (40), and (41), we have,

$$
T\left(r, f_{1}\right)=0\left((\log r) N\left(2 r, 1 / f_{1}\right)\right) \quad \text { n.e. as } r \rightarrow+\infty \text {, }
$$

and since $f_{1}$ satisfies (27), it follows now that $\log T\left(r, f_{1}\right)=o(r)$ n. e. as $r \rightarrow+\infty$. But then from (41), we have $T(r, A)=o(r)$ n. e. as $r \rightarrow+\infty$, and so it easily follows (see $[3 ; \S 4(\mathrm{~A})]$ ) that $T(r, A)=o(r)$ as $r \rightarrow+\infty$ without an exceptional set. This shows that $A(z)$ has no zeros since if $z_{0}$ is a zero of $A$, then all points $z_{0}+n w$, (for integral $n$ ) are also zeros, and the counting function for these points exceeds $c r$ as $r \rightarrow+\infty$ for some $c>0$. Hence $A$ must be the exponential of a polynomial, but being periodic will then be of the form $e^{c z+d}$ for constants $c$ and $d$. Since $T(r, A)=o(r)$ as $r \rightarrow+\infty$, we must have $c=0$, and so $A$ is a constant, contrary to hypothesis.

7. LEMMA D. Let $A(z)$ be a nonconstant entire function of period $w$, say $A(z)=B\left(e^{\alpha z}\right)$, where $B(\zeta)$ is analytic on $0<|\zeta|<\infty$, and $\alpha=2 \pi i / w$, and assume

$$
\log T(r, A)=o(r) \quad \text { as } r \rightarrow+\infty .
$$

Let $f \not 0$ be a solution of (1) which satisfies (27), and has the property that the functions $f(z)$ and $f(z+w)$ are linearly independent. Set $E(z)=f(z) f(z+w)$. 
Then

(a) $\log T(r, E)=o(r)$ as $r \rightarrow+\infty$.

(b) $E(z)^{2}$ is periodic of period $w$.

(c) Writing $E(z)^{2}=\Phi\left(e^{\alpha z}\right)$ where $\Phi(\zeta)$ is analytic on $0<|\zeta|<\infty$, then $\Phi(\zeta)$ has at most a pole at $\zeta=\infty$ (resp. $\zeta=0)$ if and only if $B(\zeta)$ has at most a pole at $\zeta=\infty$ (resp. $\zeta=0)$.

Proof. Since $E$ and $A$ are related by equation (16), we observe first that $E$ is transcendental since $A$ is transcendental. Now since $f(z)$ has property (27), it is easy to see (e.g. $[1 ;$ p. 530$])$ that $f(z+w)$ also has property $(27)$, and hence so does $E(z)$. Since $E$ satisfies (17), it now easily follows from (43) that $\log T(r, E)=o(r)$ n. e. as $r \rightarrow+\infty$, and hence (using [3; $\S 4(\mathrm{a})]$ ), we see that Part (a) holds.

For Part (b), we note that by Lemma C, we have $E(z+w) \equiv c E(z)$ for some nonzero constant $c$. Thus $E^{\prime} / E$ and $E^{\prime \prime} / E$ have period $w$, and so by (16), we obtain Part (b).

For Part (c), we set $F=E^{2}$, so $F(z)=\Phi\left(e^{\alpha z}\right)$ where $\Phi(\zeta)$ is analytic on $0<$ $|\zeta|<\infty$. From (16) it follows that $\Phi$ satisfies the equation,

$$
\alpha^{2}\left(\zeta^{2} \Phi \Phi^{\prime \prime}-(3 / 4) \zeta^{2}\left(\Phi^{\prime}\right)^{2}+\zeta \Phi \Phi^{\prime}\right)+4 B(\zeta) \Phi^{2}+K^{2} \Phi=0 .
$$

We show first that if $B(\zeta)$ has at most a pole at $\infty$, the same is true for $\Phi(\zeta)$. (The converse is obvious from (44).) If we assume the contrary, then by the Wiman-Valiron theory $[11 ;$ pp. 15, 93-111], we can write $\Phi(\zeta)$ in the form $\zeta^{m} \Psi(\zeta) u(\zeta)$, where $m$ is an integer, $\Psi(\zeta)$ is analytic on a region $|\zeta|>r_{0}$ and has a finite, nonzero limit at $\infty$, and where $u(\zeta)$ is an entire transcendental function whose maximum modulus satisfies an asymptotic relation, $\log M(r, u) \sim c_{1} r^{\sigma}$ as $r \rightarrow+\infty$, for some constants $c_{1}>0$ and $\sigma>0$. Hence, for sufficiently large $r$, if $\zeta_{r}$ denotes a point on $|\zeta|=r$ at which $|u(\zeta)|=M(r, u)$, then

$$
\left|\Phi\left(\zeta_{r}\right)\right| \geqq \exp \left(\left(c_{1} / 2\right) r^{\sigma}\right) .
$$

Writing $\zeta_{r}=r e^{i \theta_{r}}$ where $\left|\theta_{r}\right| \leqq \pi$, we set $z_{r}=\alpha^{-1}\left(\log r+\imath \theta_{r}\right)$, so $F\left(z_{r}\right)=\Phi\left(\zeta_{r}\right)$. Since $\left|z_{r}\right| \leqq 2|\alpha|^{-1} \log r$ for sufficiently large $r$, we thus have from (45),

$$
\log M\left(\left|z_{r}\right|, F\right) \geqq\left(c_{1} / 2\right) \exp \left(2^{-1}|\alpha| \sigma\left|z_{r}\right|\right),
$$

for all sufficiently large $r$. Using a standard inequality [6; p. 18, Theorem 1.6], it now follows that,

$$
\log T\left(2\left|z_{r}\right|, F\right) \geqq \log \left(c_{1} / 6\right)+2^{-1}|\alpha| \sigma\left|z_{r}\right|,
$$

for all sufficiently large $r$, which contradicts the result in Part (a) since $F=E^{2}$, and $\left|z_{r}\right| \rightarrow+\infty$ as $r \rightarrow+\infty$. This proves that $\Phi(\zeta)$ has at most a pole at $\zeta=\infty$ if the same is true of $B(\zeta)$. The analogous situation for $\zeta=0$ is proved by setting $\Phi_{1}(t)=\Phi\left(t^{-1}\right)$, and using similar reasoning at $t=\infty$. This proves Part (c). 
8. Proof of Theorem 2. We first prove Part (A). We assume that (1) possesses a solution $f \not \equiv 0$ which fails to satisfy (7), so that (27) holds. We divide the proof into two cases.

Case I: Suppose first that $f(z)$ and $f(z+w)$ (where $w=2 \pi i / \alpha$ ) are linearly independent. Then Lemma D is applicable, so if we set $E(z)=f(z) f(z+w)$, then $E(z)^{2}=\Phi\left(e^{\alpha z}\right)$, where $\Phi(\zeta)$ is analytic on $0<|\zeta|<\infty$, and $\Phi(\zeta)$ has at most poles at $\zeta=0$ and $\zeta=\infty$. Thus $\Phi(\zeta)$ is rational, and so both $\zeta \Phi^{\prime} / \Phi$ and $\zeta^{2} \Phi^{\prime \prime} / \Phi$ tend to finite limits as $\zeta \rightarrow \infty$, and as $\zeta \rightarrow 0$. Since $\Phi$ satisfies (44) for some constant $K \neq 0$, it thus follows that $4 B(\zeta)+K^{2}(\Phi(\zeta))^{-1}$ also tends to finite limits as $\zeta \rightarrow \infty$, and as $\zeta \rightarrow 0$. But by hypothesis $B(\zeta) \rightarrow \infty$ as $\zeta \rightarrow \infty$, and as $\zeta \rightarrow 0$, and hence we can conclude that $\Phi(\zeta) \rightarrow 0$ as $\zeta \rightarrow \infty$, and as $\zeta \rightarrow 0$. Thus $\Phi(\zeta)$ is analytic on the extended plane and so is a constant, which of course must be zero. Thus $f \equiv 0$ contradicting our hypothesis. Thus Case I is impossible.

Case II: We now assume $f(z)$ and $f(z+w)$ are linearly dependent. Then by Lemma $\mathrm{B}$, the solution $f(z)$ has the form (28), where conditions (i), (ii), (iii) in Lemma B are satisfied. We can assume in (28) that $d_{0}=0$ by incorporating the term $\exp \left(d_{0}\right)$ into the polynomial $\Psi$ as a constant multiplier. Since $d_{\jmath} \neq 0$ for some $j \neq 0$, we can assume in (28) that $m=\max \{j: d, \neq 0\}$ and $q=\min \left\{j: d_{,}\right.$ $\neq 0\}$ so that all the following hold in $(28)$ :

$$
d_{m} \neq 0, \quad d_{q} \neq 0, \quad m \neq 0, \quad q \neq 0, \quad \text { and } \quad q \leqq m .
$$

In (28), set $G(z)=\Psi\left(e^{\alpha z}\right)$, and,

$$
g(z)=\sum_{j=q}^{m} d_{j} e^{\alpha j z}+d z,
$$

so that $f=G e^{g}$. Since $f$ satisfies (1), we see that $A, g$, and $G$ are related by (15). We now set $H=-2 g^{\prime}\left(G^{\prime} / G\right)-\left(G^{\prime \prime} / G\right)$ so that by (15) we have,

$$
A=-\left(g^{\prime}\right)^{2}-g^{\prime \prime}+H \text {. }
$$

We observe first that since $A$ and $g$ are entire, it follows from (50) that $H$ is entire. On the other hand, since $\Psi(\zeta)$ is a polynomial, and since from (49), clearly $g^{\prime}$ and $g^{\prime \prime}$ are rational functions of $e^{\alpha z}$, we see that $H(z)$ is a rational function of $e^{\alpha z}$, say $H(z)=R\left(e^{\alpha z}\right)$, where $R(\zeta)$ is rational. We distinguish two subcases depending on whether $R \equiv 0$ or $R \not \equiv 0$.

Subcase A: If $R \equiv 0$, then $H \equiv 0$, so from (49) and (50), we have

$$
A(z)=-\left(\sum_{j=q}^{m} \alpha \jmath d_{j} e^{\alpha \jmath z}+d\right)^{2}-\sum_{j=q}^{m}(\alpha j)^{2} d_{j} e^{\alpha \jmath z} .
$$

Since $A(z)=B\left(e^{\alpha z}\right)$, we see that as rational functions of $\zeta$, we have,

$$
B(\zeta)=-\left(\sum_{j=q}^{m} \alpha j d_{j} \zeta^{\jmath}+d\right)^{2}-\sum_{j=q}^{m}(\alpha j)^{2} d_{j} \zeta^{\jmath} .
$$

Now from (48), we know $q$ and $m$ are nonzero. If both $m$ and $q$ are positive, 
it is clear from (52) that $B(\zeta)$ has no pole at $\zeta=0$, contrary to hypothesis. If both $m$ and $q$ are negative, it is clear that $B(\zeta)$ has no pole at $\zeta=\infty$, again contrary to hypothesis. If $m$ and $q$ are of opposite sign, we must have $q<0<m$, and it follows from (52) that $B(\zeta)$ has a pole at $\infty$ of order $2 m$, and a pole at zero of order $2|q|$, again contradicting the hypothesis that at least one of these poles must be of odd order. Thus Subcase A is impossible.

Subcase B: Here the rational function $R(\zeta)$ is not identically zero. Hence we can write $R=R_{1} / R_{2}$ where $R_{1}$ and $R_{2}$ are polynomials in $\zeta$, having no common factor. Since $H(z)=R\left(e^{\alpha z}\right)$ is an entire function, it is clear that $R_{2}$ can have no roots other than (possibly) zero. Hence $R_{2}(\zeta)$ is of the form $c \zeta^{m}$, and so we may write $R(\zeta)=\sum_{j=t}^{s} c_{j} \zeta^{j}$, where the $c_{j}$ are constants, where $t$ and $s$ are integers with $t \leqq s$, and where $c_{t} \neq 0$ and $c_{s} \neq 0$. Now, by definition of $H$, we have

$$
G^{\prime \prime}+2 g^{\prime} G^{\prime}=-G H,
$$

where $G(z)=\Psi\left(e^{\alpha^{2}}\right)$, and $g$ is given by (49). Now if the degree $p$ of the polynomial $\Psi(\zeta)$ is zero, then $G(z)$ is a constant, and so by (53), $H \equiv 0$. But then $R \equiv 0$, contradicting this subcase. Thus $p \geqq 1$, and we may write,

$$
\Psi(\zeta)=\sum_{j=0}^{p} a_{\jmath} \zeta^{\jmath}, \quad \text { where } \quad a_{p} \neq 0, \quad a_{0} \neq 0,
$$

and the $a$, are constants. (The fact that $a_{0} \neq 0$ follows from the fact that the roots of $\Psi$ are nonzero.) Thus from (49), (53), and (54), we have

$$
\sum_{j=1}^{p}(\alpha j)^{2} a_{j} \zeta^{j}+2\left(\sum_{j=q}^{m} \alpha j d_{j} \zeta^{\jmath}+d\right)\left(\sum_{j=1}^{p} \alpha j a_{j} \zeta^{\jmath}\right)=-\left(\sum_{j=0}^{p} a_{j} \zeta^{j}\right)\left(\sum_{j=t}^{s} c_{j} \zeta^{j}\right),
$$

as rational functions in $\zeta$. In addition, from (49) and (50), we have,

$$
B(\boldsymbol{\zeta})=-\left(\sum_{j=q}^{m} \alpha_{j} d_{j} \zeta^{\jmath}+d\right)^{2}-\left(\sum_{j=q}^{m}(\alpha j)^{2} d_{j} \zeta^{j}\right)+\sum_{j=t}^{s} c_{j} \zeta^{\jmath},
$$

as rational functions of $\zeta$. We know from (48) that $m$ and $q$ are both nonzero, and $q \leqq m$. We consider separately the three possibilities, (i) $m>0$ and $q>0$ : (ii) $m<0$ and $q<0$; (iii) $q<0<m$.

In Case (i), we have $m>0$ and $q>0$. Since also $p>0$, it is clear that when the left side of (55) is expanded in powers of $\zeta$, all terms have positive powers of $\zeta$. Now the nonzero term on the right side of (55) containing the smallest power of $\zeta$ is $-a_{0} c_{t} \zeta^{t}$, so by (55) we have $t>0$. Thus in this case, the expansion of $B(\zeta)$ given in (56) contains only nonnegative powers of $\zeta$, so that $B(\zeta)$ has no pole at $\zeta=0$ which is contrary to the hypothesis. Thus (i) cannot occur.

In Case (ii) we have $m<0$ and $q<0$. Thus, when the left side of (55) is expanded in powers of $\zeta$, all nonzero terms which appear contain a power of $\zeta$ of at most $p$. Since the right side of (55) has the nonzero term $-a_{p} c_{s} \zeta^{p+s}$, we thus have $p+s \leqq p$, so $s \leqq 0$. Since also $m<0$, we see that the expansion of $B(\zeta)$ in (56) contains only terms with nonpositive powers of $\zeta$ which contradicts the 
hypothesis that $B(\zeta)$ has a pole at $\infty$. Thus (ii) cannot occur.

In Case (iii), we have $q<0<m$. Now, in (54), let $k$ denote the smallest index $j>0$ such that $a, \neq 0$. Thus $0<k \leqq p$. Since $q<0$, the nonzero term in the expansion of the left side of (55) which has the smallest power of $\zeta$ is $2 \alpha^{2} q k d_{q} a_{k} \zeta^{k+q}$. Since the nonzero term on the right side of (55) which has the smallest power of $\zeta$ is $-a_{0} c_{t} \zeta^{t}$, we must have

$$
k+q=t \text {. }
$$

On the other hand, the nonzero terms in the expansions of the left and right sides respectively of (55), which have the largest powers of $\zeta$ are $2 \alpha^{2} m p d_{m} a_{p} \zeta^{p+m}$ and $-a_{p} c_{s} \zeta^{p+s}$ respectively, so we have $m=s$. Since $m>0$, we thus see that the nonzero term in the expansion of $B(\zeta)$ in $(56)$ which has the largest power of $\zeta$ is $-(\alpha m)^{2} d_{m}^{2} \zeta^{2 m}$. Since $q<0$ and $q<t$ (from (57), the nonzero term in $B(\zeta$ ) with the smallest power of $\zeta$ is $-(\alpha q)^{2} d_{q}^{2} \zeta^{2 q}$. Thus $B(\zeta)$ has even order poles at both $\zeta=0$ and $\zeta=\infty$ contradicting our hypothesis. Hence (iii) cannot occur, and so Subcase $B$ is impossible. Thus, our assumption of the existence of a solution which fails to satisfy (7) has led to a contradiction, proving Part (A) of Theorem 2.

For Part (B), we observe first that if $\Delta(r)$ is as in the statement, then

$$
T(2 r, A)=0(\Delta(r)) \quad \text { n.e. as } r \rightarrow+\infty \text {, }
$$

since $T(r, A)=0(r)$ as $r \rightarrow+\infty$. From Part (A), it follows that no solution $f \not \equiv 0$ of (1) can satisfy $N(r, 1 / f)=0(\Delta(r))$ n. e. as $r \rightarrow+\infty$, since such a solution, in view of the hypothesis $\log ^{+} \Delta(r)=o(r)$ n. e. as $r \rightarrow+\infty$, would satisfy (27) nearly everywhere as $r \rightarrow+\infty$. In view of $[3 ; \S 4(\mathrm{~A})]$, the solution $f$ would satisfy (27) as $r \rightarrow+\infty$ with no exceptional set, thus contradicting the conclusion (7) from Part (A). Hence, the conclusion of Part (B) now follows immediately from $[1$; Theorem 2A, p. 508].

9. Remarks. (a) For the case of an equation, $f^{\prime \prime}+B\left(e^{\alpha z}\right) f=0$, where $B(\zeta)$ is a rational function which is analytic on $0<|\zeta|<\infty$, and has even order poles at $\zeta=0$ and $\zeta=\infty$, it is possible for conclusion (7) to hold for all solutions $f \not \equiv 0$. A general class of such equations can be constructed very simply by letting $B_{1}(w)$ be any rational function of $w$, analytic on $0<|w|<\infty$, and having poles at $w=0$ and $w=\infty$, at least one of which has odd order. Then by Theorem 2, all solutions $f \not \equiv 0$ of the equation $f^{\prime \prime}+B_{1}\left(e^{2 \alpha z}\right) f=0$ satisfy (7). But this equation is the same as $f^{\prime \prime}+B\left(e^{\alpha z}\right) f=0$, where $B(\zeta)=B_{1}\left(\zeta^{2}\right)$, and clearly $B(\zeta)$ has poles of even order at both $\zeta=0$ and $\zeta=\infty$.

(b) For the case of an equation, $f^{\prime \prime}+B\left(e^{\alpha z}\right) f=0$, where $B(\zeta)$ is a rational function, analytic on $0<|\zeta|<\infty$, and has a pole at only one of the points $\zeta=0$ or $\zeta=\infty$, it is possible for all solutions $f \not \equiv 0$ to satisfy (7). Such examples are provided by the equations $f^{\prime \prime}+\left(e^{z}-K\right) f=0$, where $K$ is a constant with the property that $16 K$ is not the square of an odd, positive integer. (It was shown 
in [4] using the results in [3] that one has $\bar{\lambda}(f)=\infty$ for all solutions $f \neq 0$ of such an equation. By using the stronger results given in Lemmas B and D above, in place of the corresponding results in [3], we can obtain the stronger conclusion that (7) holds for all solutions $f \not \equiv 0$. We remark that when $16 K$ is the square of an odd, positive integer, it was shown in [4] that the equation possesses a fundamental set $\left\{f_{1}, f_{2}\right\}$ such that $\bar{\lambda}\left(f_{j}\right) \leqq 1$ for $j=1,2$.)

10. Proof of Theorem 3. We are given that $A(z)$ is a polynomial of degree $n \geqq 1$. It is shown in [12] that if $f \neq 0$ is a solution of (1) having infinitely many zeros, then there is a nonzero constant $c$ such that,

$$
\bar{N}(r, 1 / f) / r^{(n+2) / 2} \longrightarrow c \quad \text { as } r \rightarrow+\infty .
$$

(See also Kaufman [7]. Let $H_{n}$ and $P$ be as in the statement of Theorem 3 .

Assume now that all solutions $f \not \equiv 0$ of (1) have infinitely many zeros. Then for any solution $f \not \equiv 0$, the function $f^{\prime} / f$ cannot be algebraic over $H_{n}$, since in the contrary case we would have (from (8)), $T\left(r, f^{\prime} / f\right)=o\left(r^{(n+2) / 2}\right)$ as $r \rightarrow+\infty$, which would clearly contradict (59) since $c \neq 0$. Hence from [1; Theorem 4(a), p. 511], we can conclude that for the function $h(z)$ given by (3), we have that $h \not \equiv 0$ and that $h^{\prime} / h$ does not belong to $H_{n}$. Since $m\left(r, h^{\prime} / h\right)=0(\log r)$ as $r \rightarrow+\infty$, it follows that $N\left(r, h^{\prime} / h\right) \neq o\left(r^{(n+2) / 2}\right)$ as $r \rightarrow+\infty$. Since any pole of $h$ must be a pole of a coefficient of $P$, and each such coefficient satisfies (8), we have $\bar{N}(r, h)=o\left(r^{(n+2) / 2}\right)$ as $r \rightarrow+\infty$, and so $h$ cannot satisfy (9) since

$$
N\left(r, h^{\prime} / h\right)=\bar{N}(r, h)+\bar{N}(r, 1 / h) \text {. }
$$

This proves Part (A).

For Part (B), we assume that (1) possesses a solution $f_{0} \neq \equiv 0$ having only finitely many zeros. Then, $f_{0}$ must be of the form $B(z) e^{V(z)}$, where $B$ and $V$ are polynomials, with $V$ of degree $(n+2) / 2$. Hence $f_{0}$ is not algebraic over $H_{n}$, for in the contrary case, $f_{0}$ would satisfy (8), and hence $e^{V(z)}$ would satisfy (8) which is false (see $[6 ; \mathrm{p} .7]$ ). Of course, $R_{0}=-f_{0}^{\prime} / f_{0}$ is a rational function, and so belongs to $H_{n}$. Finally, for any solution $f \not \equiv 0$ of (1) which is linearly independent with $f_{0}$, we must have $\bar{\lambda}(f)=(n+2) / 2$ by [2; Theorem 1], and so $f$ satisfies (59) for some $c \neq 0$. Thus, as before, $f^{\prime} / f$ cannot be algebraic over $H_{n}$. Hence, we may apply [1; Theorem $4(\mathrm{~b})]$ to conclude that $h(z)$ given by (3) cannot be identically zero, and that $h^{\prime} / h$ belongs to $H_{n}$ if and only if $P$ has the special form given by (10). However, using (60), clearly $h^{\prime} / h$ belongs to $H_{n}$ if and only if (9) holds, which proves Part (a). The conclusions in Part (b) for solutions which are constant multiples of $f_{0}$ follow exactly as above from $[1$; Theorem 4(d)]. This proves Theorem 3.

\section{REFERENCES}

[1] S. BANK, On the value distribution theory for entire solutions of second-order linear differential equations, Proc. London Math. Soc. (3), 50 (1985), 505-534. 
[2] S. BAnk And I. LAINe, On the oscillation theory of $f^{\prime \prime}+A f=0$ where A is entire, Trans. Amer. Math. Soc. 273 (1982), 351-363.

[3] S. BANK AND I. LAINE, Representations of solutions of periodic second-order linear differential equations, J. reine angew. Math. 344 (1983) , 1-21.

[4] S. BANK, I. LAINE AND J. LANGLEY, On the frequency of zeros of solutions of second-order linear differential equations, Resultate der Math. (To appear).

[5] M. FREI, Über die subnormalen Lösungen der Differentialgleichung $w^{\prime \prime}+e^{-z} w^{\prime}+$ (const.) $w=0$. Comm. Math. Helv. 36 (1962), 1-8.

[6] W. Hayman, Meromorphic Functions. Clarendon Press, Oxford (1964).

[7] R. KAUFMAN, Riccati equations, zeros, and independence, J. reine angew. Math. 345 (1983), 63-68.

[8] R. Nevanlinna, Le Théorème de Picard-Borel et la Théorie des Fonctions Méromorphes, Gauthier-Villars, Paris, 1929.

[9] M. OzawA, On a solution of $w^{\prime \prime}+e^{-z} w^{\prime}+(a z+b) w=0$. Kodai Math. J. 3 (1980), 295-309.

[10] S. Saks And A. Zygmund, Analytic Functions, Monografie Mat. (Engl. Transl.), Tom 28, Warsaw, 1952.

[11] G. VALIRON, Lectures on the General Theory of Integral Functions, Chelsea Publ. Co., New York, 1949.

[12] H. Wiтtich, Eindeutige Lösungen der Differentialgleichung $w^{\prime}=R(z, w)$, Math. Zeit. 74 (1960), 278-288.

[13] H. Wiтtich, Subnormale Losungen der Differentialgleichung $w^{\prime \prime}+p\left(e^{z}\right) w^{\prime}+q\left(e^{z}\right) w$ $=0$. Nagoya Math. J. 30 (1967), 29-37.

Department of Mathematics

UNIVERSITY OF ILLINOIS

1409 W. Green Street

URBANA, ILLINOIS 61801

U.S.A. 